\title{
Curcumin modulates covalent histone modification and TIMP1 gene activation to protect against vascular injury in a hypertension rat model
}

\author{
JUN HU, TINGTING SHEN，JUN XIE，SIYANG WANG，YUE HE and FU ZHU
}

Xuhui Central Hospital, Shanghai Clinical Research Center, China Academy of Sciences, Shanghai 200031, P.R. China

Received August 21, 2015; Accepted January 6, 2017

DOI: 10.3892/etm.2017.5318

\begin{abstract}
Hypertension is a leading risk factor for morbidity and mortality. Previous studies have reported that curcumin has anti-oxidation and anti-aging effects and inhibits histone deacetylase activity. However, it is still unclear whether curcumin could protect against vascular injury induced by hypertension. Thus, the current study examined the therapeutic effects and mechanism of curcumin on vascular injury induced by hypertension in spontaneous hypertensive rats (SHRs). The present study revealed that curcumin may improve vascular structure and attenuate coronary artery pathology. Moderate doses ( $\sim 50 \mathrm{mg})$ of curcumin were most effective in treating coronary artery injury in SHRs. Moreover, the results of immunohistochemical analysis indicated that the expression levels of histone deacetylase 1 (HDAC1), matrix metalloproteinase-2 (MMP-2) and transforming growth factor $\beta$ (TGF $\beta$ ) decreased in the curcumin treatment group, compared with the non-treated group or the negative control group. However, the expression of tissue inhibitor of metalloproteinase 1 (TIMP1) did not visibly decrease. Furthermore, chromatin immunoprecipitation results suggested that curcumin was capable of promoting the transcription activation of TIMP1 through suppressing HDAC1 expression and increasing histone $\mathrm{H} 3$ acetylation at the TIMP1 promoter region in SHRs. In conclusion, curcumin could relieve extracellular matrix degradation and interstitial fibrosis induced by hypertension, and lower blood pressure. It could also serve a function in improving vascular structure through inhibiting the expression of HDAC1, thereby promoting TIMP1 transcription activation and suppressing the expression of MMP-2 and TGF $\beta$.
\end{abstract}

\section{Introduction}

Hypertension is a leading risk factor for morbidity and mortality $(1,2)$. The elevation of blood pressure is accompanied

Correspondence to: Professor Fu Zhu, Xuhui Central Hospital, Shanghai Clinical Research Center, China Academy of Sciences, 966 Huai Hai Zhong Road, Shanghai 200031, P.R. China

E-mail: fuzhu2015@126.com

Key words: hypertension, spontaneous hypertensive rats, curcumin, coronary artery, epigenetics by increased reactive oxygen species production, decreased nitric oxide bioavailability and decreased antioxidant capacity $(2,3)$. These factors may contribute to the elevation of arterial blood pressure as well as end organ damage in hypertension (2,3). Furthermore, matrix metalloproteinases (MMPs) may be involved in end organ damage in hypertension (2). MMPs are a family of proteases that degrade extracellular matrix (ECM) proteins (4,5). They are produced as pro-MMPs, which are then cleaved into active MMPs $(4,5)$. Classes of MMP include collagenases, gelatinases, stromelysins, matrilysins and membrane-types (4). MMP-2 (gelatinase A) and MMP-9 (gelatinase B) are expressed in the uterus of humans and bovines, as well as other mammals, and they serve a role in animal and human endometrial tissue remodeling during the estrous and menstrual cycles, and in pregnancy (4).

Curcumin (diferuloylmethane) is a component of turmeric $(2-5 \%, w / w)$ a spice that gives the yellow color to curry powder. In traditional Ayurveda medicine, turmeric has been described as a potent anti-inflammatory agent (6). The suppression of cellular transformation, proliferation, invasion, angiogenesis and metastasis of tumors by curcumin has been reported (6). On the other hand, a low concentration of curcumin (500 nM) results in a neurological protective effect, through increasing the proliferation of neural stem cells in the hippocampus (7). It has been shown that curcumin protects the vascular endothelium, through the production of endothelial heme-oxigenase, while in Alzheimer's disease it counteracts $\beta$-amyloid-induced oxidative stress (7). Some of these observations can be explained by the activating and inhibitory actions of curcumin on a number of key signaling pathways, including those of MAP kinase, protein kinase $\mathrm{C}$ and brain-derived neurotrophic factor. Given its activity on histone deacetylases (HDACs) (7), curcumin may also be considered an epigenetic drug. Furthermore, curcumin can partially protect against fructose-induced impairment in vascular contractility via an antioxidant effect and reduction of elevated intracellular calcium (8).

HDACs regulate the expression of cancer-associated genes, which makes them promising targets for the development of novel cancer drugs (9). Several HDAC inhibitors, including suberanilohydroxamic acid, valproic acid and trichostatin A, have been used within a clinical setting for the treatment of cancer, or are undergoing clinical trials (9). The HDAC family contains 18 proteins, which are grouped into four classes (I-IV) based on their structure and homology. Classes I, II 
and IV contain 11 family members and they are known as the classical HDACs, while the seven class III family members are referred to as sirtuins (9).

The aim of the current study is to examine the therapeutic effects and mechanism of curcumin on vascular injury induced by hypertension in spontaneous hypertensive rats (SHRs). The protective effects of curcumin against hypertension-caused injury by regulating gene expression levels of MMP-2, HDAC1, tissue inhibitor of metalloproteinase 1 (TIMP1) and transforming growth factor $\beta$ (TGF $\beta$ ) are investigated.

\section{Materials and methods}

Animals. A total of 42 male SHRs (weight, $200 \mathrm{~g}$ ), aged 8-10 weeks, were obtained from the Shanghai Research Center for Model Organisms (Shanghai, China). This study was approved (permit no. SRCMR20130016) by the Animal Ethics Committee of Shanghai Research Center for Model Organisms, and the experimental protocols were in compliance with the Experimental Animal Regulations of the Ministry of Science and Technology (Beijing, China). All rats were maintained in colonies of 2 per cage for 14 days, and housed in a temperature-controlled room under a standard light-dark cycle, with free access to food and water. The animals were divided into 7 groups as follows: Blank control group $(n=6)$ without any treatment, negative control group $(n=6)$ that received saline only (by intraperitoneal injection) and 5 experimental groups $(n=6$ in each) that were administered curcumin by a single intraperitoneal injection $(200 \mu \mathrm{l})$ every 2 days, for a total of 56 days, in concentrations of 25, 50, 100, 200 and $400 \mathrm{mg} / \mathrm{kg}$ body weight. Curcumin was obtained from Shanghai Sinopharm Chemical Reagent Company, Ltd. (Shanghai, China). A total of 8 weeks following the start of treatment, the rats (weight $\sim 200 \mathrm{~g}$ ) were anesthetized with $1.5 \%$ Nembutal (30 mg/kg; Sigma-Aldrich; Merck KGaA, Darmstadt, Germany) administered by intraperitoneal injection. Following anesthesia the rats were sacrificed by cervical dislocation and additional experiments were conducted.

Histopathological analysis. For histopathological analysis, coronary artery tissues were stained with hematoxylin and eosin (H\&E) and Massion stains. Briefly, all fresh tissues were washed three times with PBS and fixed with $4 \%$ paraformaldehyde (Sigma-Aldrich; Merck KGaA) for 30 min, dehydrated through a graded series of ethanol, vitrified in xylene and embedded in paraffin. Next, serial $6-\mu \mathrm{m}$ sections were cut and stained with H\&E. The Massion stain was performed according to the manufacturer's protocol (Sigma-Aldrich; Merck KGaA).

Immunohistochemistry (IHC) analysis. Briefly, all steps were carried out as previously described (10). The animals were sacrificed by cervical dislocation and the aorta was subsequently isolated from the root to the abdominal aortic bifurcation for disconnection. The coronary artery was harvested, cut longitudinally and washed with saline. All fresh coronary artery tissues were washed three times with PBS and fixed with $4 \%$ paraformaldehyde (Sigma-Aldrich; Merck $\mathrm{KGaA}$ ) for $30 \mathrm{~min}$, dehydrated through a graded series of ethanol (cat. no. 10009218), vitrified in xylene (cat. no. 10023418) and embedded in paraffin (cat. no. 69018961) (all Shanghai Sinopharm Chemical Reagent Company, Ltd.). Next, serial 6- $\mu$ m-thick sections were cut and rinsed with 3\% PBS (Sigma-Aldrich; Merck KGaA). Antigen retrieval was performed at $100^{\circ} \mathrm{C}$ using an antigen repairing solution Improved Citrate Antigen Retrieval solution (cat. no. P0083; Beyotime Institute of Biotechnology, Haimen, China). Rabbit anti-human HDAC1 (cat. no. 34589), MMP-2 (cat. no. 87809) and TIMP1 (cat. no. 8946), rabbit anti-histone H3 acetyl (cat. no. 9927) and anti-TGFb (cat. no. 3711) primary antibodies (all 1:200; Cell Signaling Technology, Inc., Danvers, MA, USA) were added and the sections were incubated for $60 \mathrm{~min}$ at room temperature, then horseradish peroxidase-conjugated secondary antibody (cat. no. sc-2768; 1:200; Santa Cruz Biotechnology, Inc., Dallas, TX, USA) was added and the sections were incubated for $60 \mathrm{~min}$ at room temperature. Finally, a VECTASTAIN Elite ABC kit (cat. no. PK-6100; Vector Laboratories, Inc., Burlingame, CA, USA) was used for the color reaction. Meanwhile, PBS ( $\mathrm{pH} 7.4$ ) was used as a negative control in the place of the first antibody. Five random fields of each tissue section were observed (magnification, x200) and analyzed using Image-Pro Plus version 6.0 software (Media Cybernetics, Inc., Rockville, MD, USA). ImageJ 1.42q software (National Institutes of Health, Bethesda, MD, USA) was used to analyze the area of the positively stained cells.

Chromatin immunoprecipitation (ChIP) assays. ChIP experiments were performed on coronary artery tissue sections, which were prepared as described above, using rabbit anti-human HDAC1 (cat. no. 34589) and rabbit anti-histone H3 acetyl (cat. no. 9927) primary antibodies (both 1:100; Cell Signaling Technology, Inc.), and normal rabbit immunoglobulin G (cat. no. 12-370; Upstate Biotechnology; Merck Millipore, Billerica, MA, USA) as a negative control. All steps were carried out as previously described (11). In brief, the cells were fixed with $1 \%$ formaldehyde (Sigma-Aldrich; Merck KGaA) for $30 \mathrm{~min}$ at $37^{\circ} \mathrm{C}$, then quenched with $125 \mathrm{mM}$ glycine for $10 \mathrm{~min}$ at room temperature to form DNA-protein cross-links. Samples were sonicated on ice until chromatin fragments were 200-1,000 bp in size, then incubated with antibodies at $4^{\circ} \mathrm{C}$ overnight. PCR amplification was performed under the following conditions: Thirty-three cycles in total, consisting of denaturation at $95^{\circ} \mathrm{C}$ for $30 \mathrm{sec}$, annealing at $55^{\circ} \mathrm{C}$ for $30 \mathrm{sec}$ and extension at $72^{\circ} \mathrm{C}$ for $30 \mathrm{sec}$.

Statistical analysis. Each experiment was performed at least three times. Data are shown as the mean \pm standard error and were analyzed using the Student's t-test by GraphPad Prism software, version 5.00 (GraphPad Software, Inc., La Jolla, CA, USA). $\mathrm{P}<0.05$ was considered to indicate a statistically significant difference.

\section{Results}

Effect of curcumin on coronary artery structure and blood pressure in SHRs. At 8 weeks after treatment, the coronary artery structure, blood pressure and pulse pressure in each SHR group were determined. There was no significant difference in the coronary artery external diameter between the non-treatment group and the saline group or the curcumin 

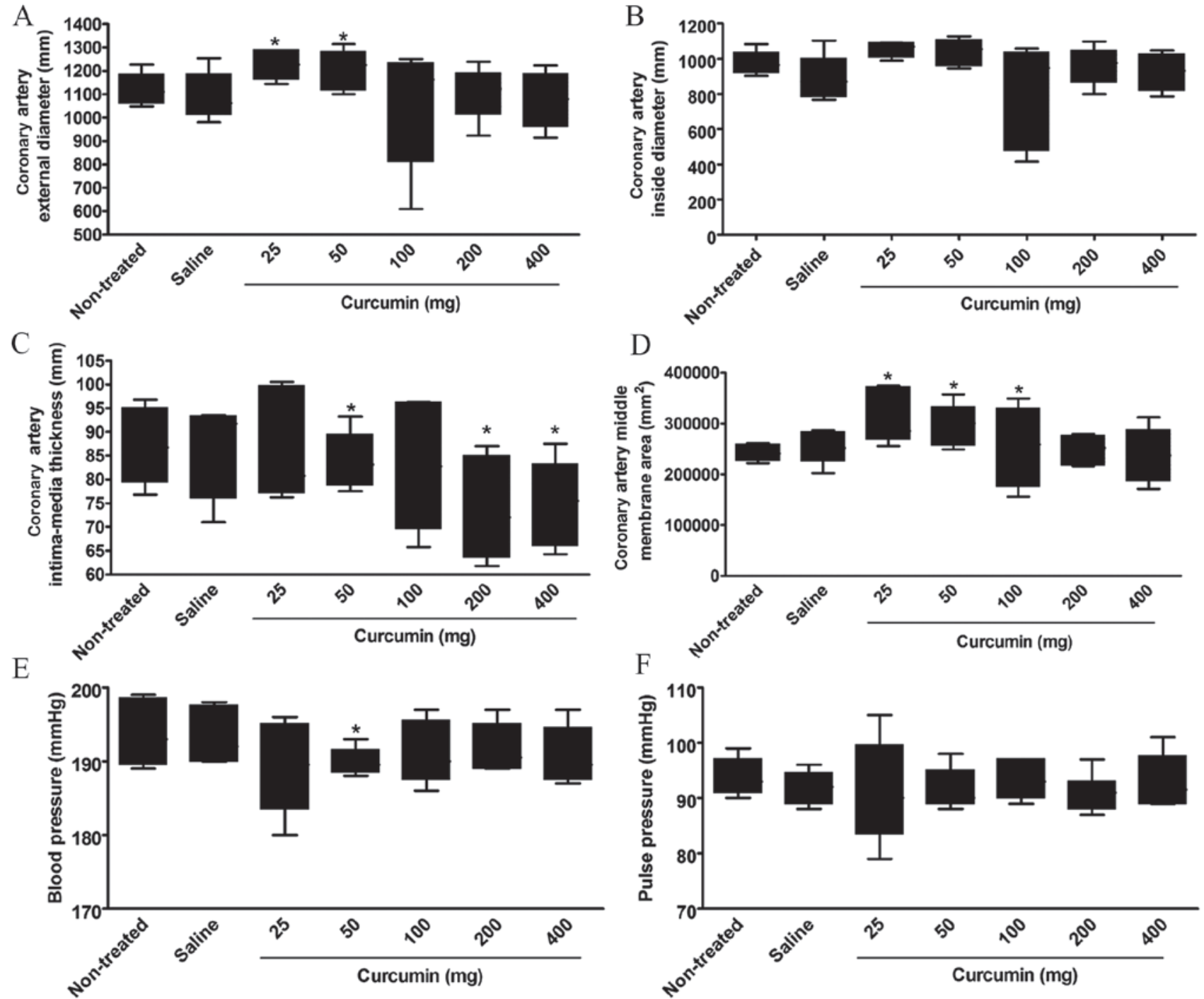

Figure 1. Effect of curcumin treatment on coronary artery structure and blood pressure in SHRs. (A) External diameter of coronary artery in SHR rats of each group. (B) Inside diameter of coronary artery in SHR rats of each group. (C) Intima-media thickness of coronary artery in SHR rats of each group. (D) Middle membrane area of coronary artery in SHR rats of each group. (E) Blood pressure in coronary artery in SHR rats of each group. (F) Pulse pressure in coronary artery in SHR rats of each group. ${ }^{*} \mathrm{P}<0.05$ vs. non-treated, $\mathrm{n}=6$ in each group. SHR, spontaneous hypertensive rat.

treatment groups with dosages of 100,200 or $400 \mathrm{mg}$. However, the external diameter of the coronary artery in the curcumin treatment groups with dosages of 25 or $50 \mathrm{mg}$ was significantly increased as compared with the non-treatment group $(\mathrm{P}<0.05$; Fig. 1A, Table I).

No significant difference was found in the coronary artery inside diameter between the groups at 8 weeks after treatment (Fig. 1B, Table I).

The coronary artery intima-media thickness significantly decreased in the curcumin treatment groups with dosages of 50, 200 and $400 \mathrm{mg}$ at 8 weeks after treatment as compared with the non-treatment group $(\mathrm{P}<0.05)$. However, no significant decrease was found in the curcumin treatment groups with dosages of 25 or $100 \mathrm{mg}$ (Fig. 1C, Table I).

The coronary artery middle membrane area significantly increased in the curcumin treatment groups with dosages of 25,50 and $100 \mathrm{mg}$, as compared with the non-treated group $(\mathrm{P}<0.05)$. However, no significant difference was found between the curcumin treatment groups with dosages of 200 or $400 \mathrm{mg}$ and the non-treated group (Fig. 1D, Table I).
A significant increase in blood pressure was only found in the curcumin treatment group with a dosage of $50 \mathrm{mg}$ as compared with the non-treated group $(\mathrm{P}<0.05)$. All other dosages showed no significant difference in blood pressure compared with the non-treated group (Fig. 1E, Table I).

No significant difference was found in the pulse pressure between the groups at 8 weeks after treatment (Fig. 1F, Table I).

Effect of curcumin on coronary artery pathology in SHRs. Hypertension can cause coronary artery stenosis and blood flow resistance, and promote inflammatory cell hyperplasia and atherosclerosis plaque formation. Consistent with this, the coronary arteries of SHRs demonstrated vascular adventitial fibrosis, smooth muscle cell hyperplasia, coronary artery endometrial glass-like lesions and vascular endothelial cell injury and hyperplasia (Fig. 2). However, at 8 weeks after treatment, in the curcumin treatment groups with dosages of 25 , 50 and $100 \mathrm{mg}, \mathrm{H} \& \mathrm{E}$ (Fig. 2A) and Massion (Fig. 2B) stains revealed that coronary artery inflammation in vascular endothelial cells had decreased and the apoptosis of endothelial 

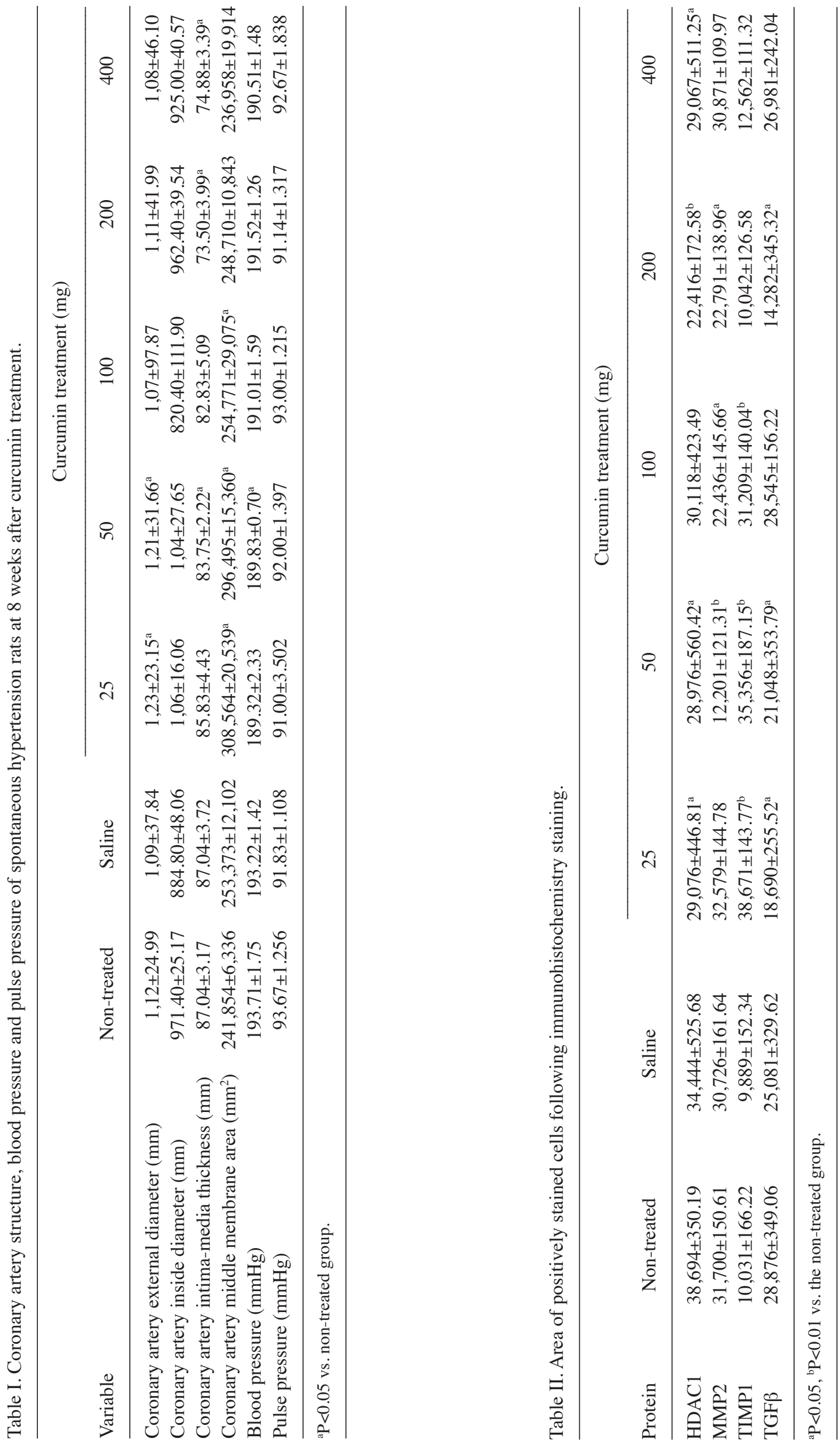


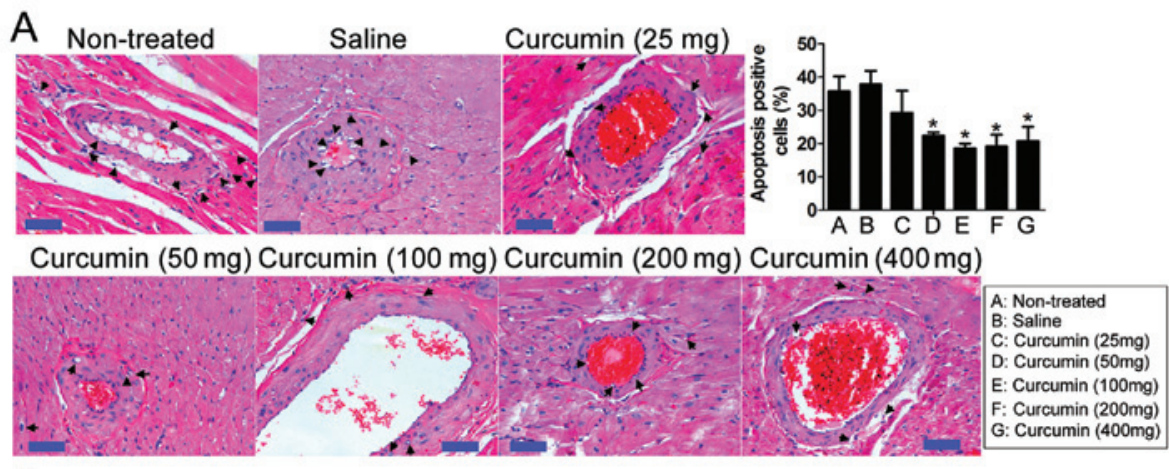

B
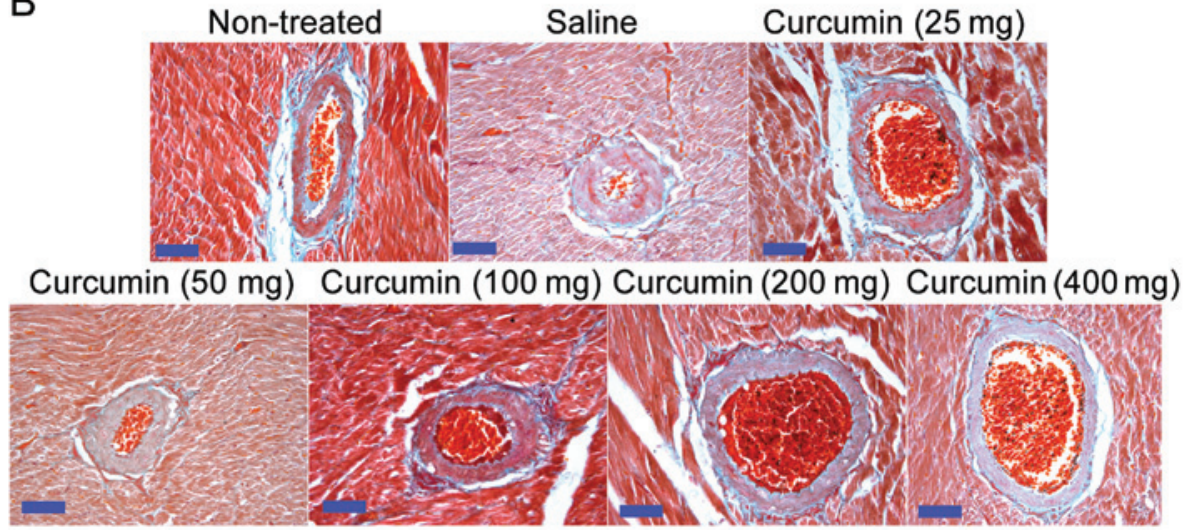

Figure 2. Effect of curcumin on coronary artery pathology in spontaneous hypertensive rats. (A) Pathological examination using hematoxylin and eosin staining. The coronary arteries of spontaneous hypotensive rats demonstrated vascular adventitial fibrosis, smooth muscle cell hyperplasia, coronary artery endometrial glass-like lesions, and vascular endothelial cell injury and hyperplasia. In the curcumin treatment groups, the coronary artery inflammation in vascular endothelial cells decreased and the apoptosis of endothelial cells increased. The black arrows indicate apoptotic cells. ${ }^{*} \mathrm{P}<0.05$ vs. non-treated cells (B) Vascular structure analysis using Massion staining. In the curcumin treatment groups, the inner wall of the blood vessel had thickened and atherosclerotic plaques were reduced. There was also a reduction in the accumulation of perivascular collagen and fibrosis. Scale bar, $30 \mu$ m; magnification, $\mathrm{x} 200$.

cells had decreased. Following H\&E staining, apoptotic cells exhibit a clear nuclear contraction and cytoplasmic expansion phenotype. The proportion of apoptotic cells to the total cell number was calculated and the statistical results indicated that curcumin treatment $(50-400 \mathrm{mg})$ significantly decreased the apoptotic rate of vascular endothelial cells and smooth muscle cells in rats $(\mathrm{P}<0.05)$. In each assay, the inner wall of the blood vessel had thickened and atherosclerotic plaques were reduced. In addition, the accumulation of perivascular collagen and fibrosis was reduced.

Effect of curcumin on expression levels of HDAC1, MMP-2, TIMPI and TGF $\beta$ in the coronary arteries of SHRs. ImageJ $1.42 \mathrm{q}$ software was used to analyze the area of positive cells on IHC staining (Table II and Fig. 3). IHC analysis indicated that at 8 weeks following treatment with $25 \mathrm{mg}$ curcumin, the expression of HDAC1 and TGF $\beta$ were significantly lower compared with the non-treated group $(\mathrm{P}<0.05)$. However, the expression of TIMP1 was significantly higher in the curcumin treatment groups $(25-100 \mathrm{mg})$ compared with the non-treated group $(\mathrm{P}<0.01)$. At 8 weeks following treatment with $50 \mathrm{mg}$ curcumin, the expression of HDAC1 $(\mathrm{P}<0.05), \mathrm{MMP} 2(\mathrm{P}<0.01)$ and TGF $\beta(\mathrm{P}<0.05)$ were all significantly lower compared with the non-treated group, however the expression of TIMP1 was significantly higher $(\mathrm{P}<0.01)$. At 8 weeks following treatment with $100 \mathrm{mg}$ curcumin, only the expression of MMP2 was significantly decreased compared with the non-treated group
$(\mathrm{P}<0.05)$, however, the expression of TIMP1 was significantly higher $(\mathrm{P}<0.01)$. At 8 weeks following treatment with $200 \mathrm{mg}$ curcumin, the expression of MMP2 $(\mathrm{P}<0.05)$, TGF $\beta(\mathrm{P}<0.05)$ and HDAC1 $(\mathrm{P}<0.01)$ were significantly lower compared with the non-treated group, however, the expression of TIMP1 was not significantly higher. At 8 weeks following treatment with $400 \mathrm{mg}$ curcumin, only the expression of HDAC1 was significantly lower in the curcumin treatment groups than in the non-treated group $(\mathrm{P}<0.05)$.

Effect of curcumin on the transcriptional activity of TIMPI genes. A ChIP assay was performed to evaluate histone $\mathrm{H} 3$ acetylation levels of TIMP1 promoters in each SHR group. At 8 weeks after treatment, the curcumin treatment groups with dosages of 50-400 mg demonstrated significantly higher levels of acetylation of histone $\mathrm{H} 3$ in the TIMP1 promoter regions as compared with the non-treated group $(\mathrm{P}<0.01, \mathrm{P}<0.05$; Fig. 4A). However, the acetylation levels of histone $\mathrm{H} 3$ in the TIMP1 promoter regions did not significantly increase in the saline group or in the curcumin group with a dosage of $25 \mathrm{mg}$ as compared with the non-treated group.

In addition, a ChIP assay was performed to evaluate HDAC1 levels at the TIMP1 promoter regions in SHRs. At 8 weeks after treatment, the levels of HDAC1 at the TIMP1 promoter regions had not significantly decreased in the saline group or the curcumin group with a dosage of $25 \mathrm{mg}$, as compared with the non-treated group. However, in the curcumin treatment 


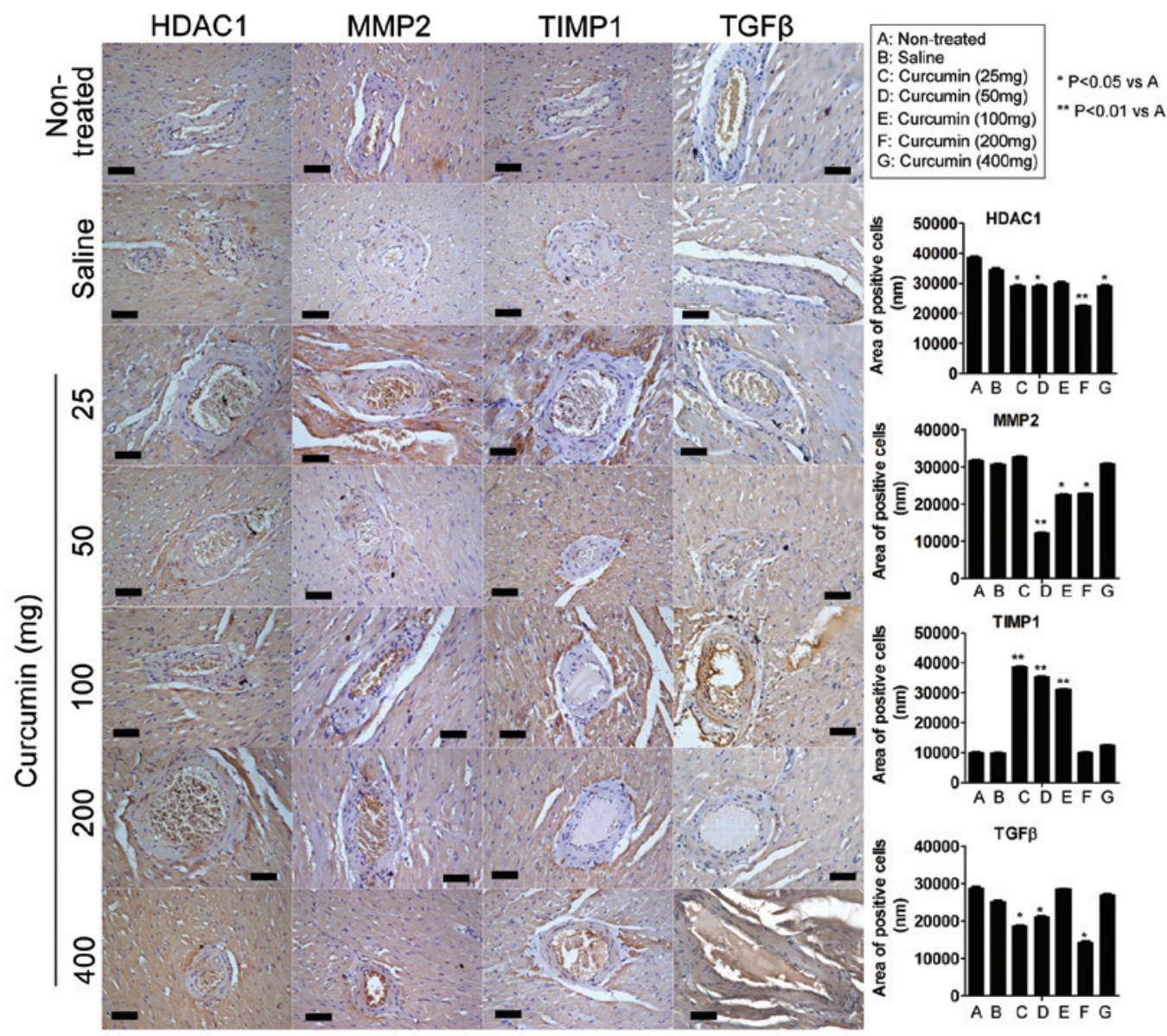

Figure 3. Effect of curcumin on protein expression in the coronary arteries of spontaneous hypertensive rats. The expression levels of HDAC1, MMP-2, TIMP1 and TGF $\beta$ proteins were determined by immunohistochemical staining. At 8 weeks following treatment, immunohistochemistry analysis indicated that the expression of HDAC1, MMP-2 and TGF $\beta$ was significantly lower in certain curcumin treatment groups when compared with the non-treated group. Certain curcumin treatment groups demonstrated significantly higher levels of TIMP1 expression compared with the non-treated group. Scale bar, $30 \mu \mathrm{m}$; magnification, x200. HDAC1, histone deacetylase 1; MMP-2, matrix metalloproteinase-2; TIMP1, tissue inhibitor of metalloproteinase 1; TGF $\beta$, transforming growth factor $\beta$.

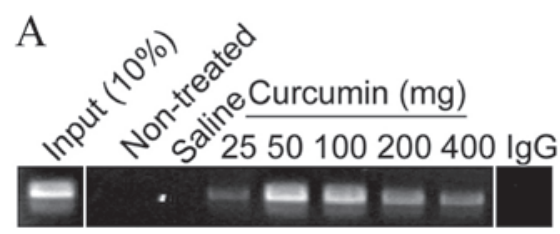

IP: anti-H3-Ac PCR: TIMP1

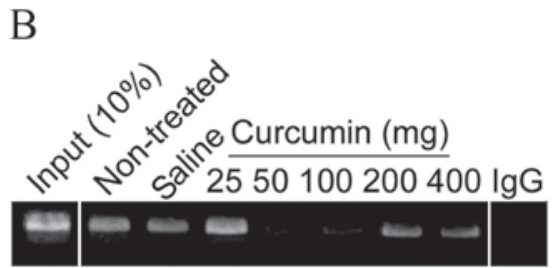

IP: anti-HDAC1 PCR: TIMP1
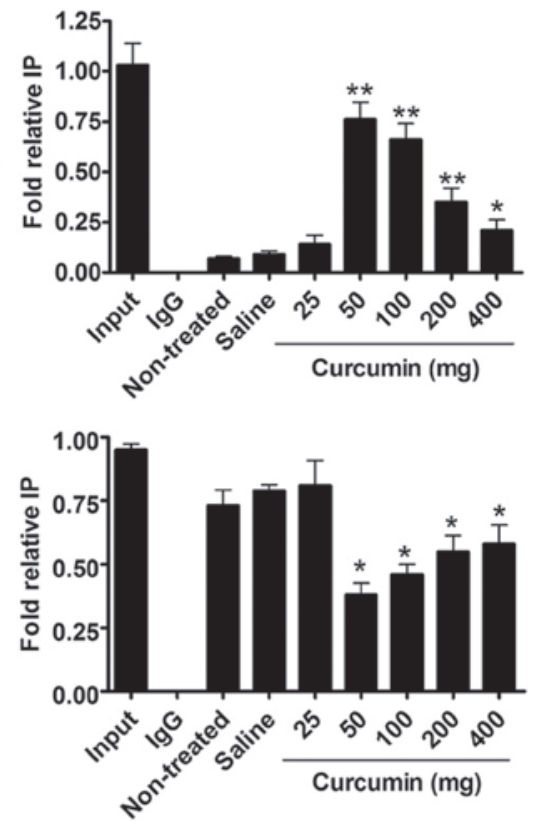

Figure 4. Effect of curcumin on the histone H3 acetylation levels of TIMP1 promoters in spontaneous hypertensive rats. (A) PCR-amplified genomic DNA obtained following ChIP with an anti-Ac-H3 antibody. (B) PCR-amplified genomic DNA obtained following ChIP with an anti-HDAC1 antibody. ${ }^{*} \mathrm{P}<0.05$ vs. non-treated, ${ }^{* *} \mathrm{P}<0.01$ vs. non-treated, $\mathrm{n}=6$ in each group. ChIP, chromatin immunoprecipitation; IP, immunoprecipitation; PCR, polymerase chain reaction; anti-Ac-H3, anti-acetylated histone $\mathrm{H} 3$. 
groups with dosages of 50-400 mg), the levels of HDAC1 at the TIMP1 promoter regions had significantly decreased as compared with the non-treated group $(\mathrm{P}<0.05$; Fig. 4B). These findings suggested that curcumin was capable of increasing the activation of the TIMP1 promoter through suppressing $\mathrm{HDAC} 1$ expression and increasing histone $\mathrm{H} 3$ acetylation.

\section{Discussion}

Hypertension is a major risk factor for cardiovascular events (12). In addition to the alterations triggered by chronic hypertension on cardiomyocytes, important modifications occur in the cardiac ECM integrity during the progression of left-ventricular hypertrophy (12). A previous study demonstrated that perturbed ECM synthesis and degradation were the primary contributors to cardiac remodeling observed in hypertensive heart disease and were caused by an imbalance in the ratio of MMPs and their inhibitors, TIMPs (13). MMP-2, MMP-9 and TIMP1 expression levels were previously demonstrated to be enhanced in the myocardium of Dahl salt-sensitive rats during the transition to congestive heart failure $(13,14)$. Moreover, MMP inhibition results in the remodeling of vascular, glomerular and tubulointerstitial spaces by altering the turnover of ECM proteins (14). A previous study also indicated that MMP-2 played a key role in hypertensive cardiac remodeling and in other cardiovascular disorders (12).

In the current study, it was revealed that curcumin effectively improved vascular structure and reduced pathological damage to the coronary arteries. Although a range of concentrations of curcumin were useful for the treatment of coronary artery injury in SHR rats, the best results were observed with moderate doses (approximately $50 \mathrm{mg} / \mathrm{kg}$ body weight). Therefore, we speculated that this dosage of curcumin had a potential therapeutic effect.

In previous studies, curcumin could inhibit the activity of HDACs (7). In the current study, the expression levels of HDAC1, MMP-2, TIMP1 and TGF $\beta$ proteins were determined after treatment with curcumin in dosages from 25 to $400 \mathrm{mg}$. The results indicated that the expression levels of HDAC1, MMP-2 and TGF $\beta$ decreased in the curcumin treatment groups, as compared with the non-treated group or the negative control group. However, the expression of TIMP1 did not decrease in the curcumin treatment groups.

The main function of HDACs is to remove acetyl groups from the $\mathrm{N}$-acetyl lysines on a histone, modify the chromatin structure and modulate gene transcription (9). The current study investigated the regulation mechanism of curcumin on covalent histone modifications and the expression levels of HDAC1. It was found that curcumin could suppress HDAC1 expression and increase histone $\mathrm{H} 3$ acetylation at the TIMP1 promoter region in SHR rats, which may promote TIMP1 transcription activation.

In conclusion, curcumin could relieve extracellular matrix degradation and interstitial fibrosis induced by hypertension, elevate blood pressure and improve vascular structure through inhibiting the expression of HDAC1, promote TIMP1 transcription activation and inhibit the expression of MMP-2 and TGF $\beta$. However, the results of the present study were conducted in an animal model, so are not necessarily wholly representative of the effect curcumin may have in humans. In the present study, the epigenetic mechanisms underlying the effect of curcumin on the regulation of vascular wall collagen was preliminarily demonstrated. However, further research is required to clarify how curcumin induces histone acetylation and the deacetylation of other proteins, as this was not investigated in depth within the present study. Further research may also focus on the effects of curcumin on the regulation of lipid metabolism and its protein interaction networks.

\section{Acknowledgements}

The present study was supported by a grant from the Shanghai Municipal Health Bureau Fund (grant no. 20114312), awarded to Professor Jun Hu (Xuhui Central Hospital, Shanghai, China), and by a grant from the Shanghai Natural Science Fund (grant no. 17ZR1426800), awarded to Professor Fu Zhu (Xuhui Central Hospital).

\section{References}

1. Kakar P and Lip GY: Towards understanding the aetiology and pathophysiology of human hypertension: Where are we now? J Hum Hypertens 20: 833-836, 2006.

2. Duansak N and Schmid-Schönbein GW: The oxygen free radicals control MMP-9 and transcription factors expression in the spontaneously hypertensive rat. Microvasc Res 90: 154-161, 2013.

3. Sedeek M, Hébert RL, Kennedy CR, Burns KD and Touyz RM: Molecular mechanisms of hypertension: Role of Nox family NADPH oxidases. Curr Opin Nephrol Hypertens 18: 122-127, 2009.

4. Li W, Mata KM, Mazzuca MQ and Khalil RA: Altered matrix metalloproteinase-2 and -9 expression/activity links placental ischemia and anti-angiogenic sFlt-1 to uteroplacental and vascular remodeling and collagen deposition in hypertensive pregnancy. Biochem Pharmacol 89: 370-385, 2014.

5. Luizon MR, Palei AC, Sandrim VC, Amaral LM, Machado JS, Lacchini R, Cavalli RC, Duarte G and Tanus-Santos JE: Tissue inhibitor of matrix metalloproteinase-1 polymorphism, plasma TIMP-1 levels, and antihypertensive therapy responsiveness in hypertensive disorders of pregnancy. Pharmacogenomics J 14: 535-541, 2014.

6. Aggarwal BB: Prostate cancer and curcumin: Add spice to your life. Cancer Biol Ther 7: 1436-1440, 2008.

7. Panighini A, Duranti E, Santini F, Maffei M, Pizzorusso T, Funel N, Taddei S, Bernardini N, Ippolito C, Virdis A and Costa M: Vascular dysfunction in a mouse model of rett syndrome and effects of curcumin treatment. PLoS One 8: e64863, 2013.

8. Mahmoud MF and El Bassossy HM: Curcumin attenuates fructose-induced vascular dysfunction of isolated rat thoracic aorta rings. Pharm Biol 52: 972-977, 2014.

9. Song C, Zhu S, Wu C and Kang J: Histone deacetylase (HDAC) 10 suppresses cervical cancer metastasis through inhibition of matrix metalloproteinase (MMP) 2 and 9 expression. J Biol Chem 288: 28021-28033, 2013.

10. Shen DZ, Xin SL, Chen C and Liu T: Effect of atorvastatin on expression of TLR4 and NF- $\mathrm{B}$ p 65 in atherosclerotic rabbits. Asian Pac J Trop Med 6: 493-496, 2013.

11. Liu T, Huang Y, Huang Q, Jiang L, Guo L and Liu Z: Use of human amniotic epithelial cells as a feeder layer to support undifferentiated growth of mouse spermatogonial stem cells via epigenetic regulation of the Nanog and Oct-4 promoters. Acta Biol Hung 63: 167-179, 2012.

12. Rizzi E, Guimaraes DA, Ceron CS, Prado CM, Pinheiro LC, Martins-OliveiraA,GerlachRFandTanus-SantosJE: $\beta 1$-Adrenergic blockers exert antioxidant effects, reduce matrix metalloproteinase activity and improve renovascular hypertension-induced cardiac hypertrophy. Free Radic Biol Med 73: 308-317, 2014

13. Kinoshita T, Ishikawa Y, Arita M, Akishima-Fukasawa Y, Fujita K, Inomata N, Suzuki T, Namiki A, Mikami T and Ikeda T: Antifibrotic response of cardiac fibroblasts in hypertensive hearts through enhanced TIMP-1 expression by basic fibroblast growth factor. Cardiovasc Pathol 23: 92-100, 2014.

14. Pushpakumar SB, Kundu S, Metreveli N, Tyagi SC and Sen U: Matrix metalloproteinase inhibition mitigates renovascular remodeling in salt-sensitive hypertension. Physiol Rep 1: e00063, 2013. 